\title{
Stability Analysis of the Continuous-Conduction- Mode Buck Converter Via Filippov's Method
}

\author{
Damian Giaouris, Member, IEEE, Soumitro Banerjee, Senior Member, IEEE, Bashar Zahawi, Senior Member, IEEE, \\ and Volker Pickert, Member, IEEE
}

\begin{abstract}
To study the stability of a nominal cyclic steady state in power electronic converters, it is necessary to obtain a linearization around the periodic orbit. In many past studies, this was achieved by explicitly deriving the Poincaré map that describes the evolution of the state from one clock instant to the next and then locally linearizing the map at the fixed point. However, in many converters, the map cannot be derived in closed form, and therefore this approach cannot directly be applied. Alternatively, the orbital stability can be worked out by studying the evolution of perturbations about a nominal periodic orbit, and some studies along this line have also been reported. In this paper, we show that Filippov's method-which has commonly been applied to mechanical switching systems-can be used fruitfully in power electronic circuits to achieve the same end by describing the behavior of the system during the switchings. By combining this and the Floquet theory, it is possible to describe the stability of power electronic converters. We demonstrate the method using the example of a voltage-mode-controlled buck converter operating in continuous conduction mode. We find that the stability of a converter is strongly dependent upon the so-called saltation matrix-the state transition matrix relating the state just after the switching to that just before. We show that the Filippov approach, especially the structure of the saltation matrix, offers some additional insights on issues related to the stability of the orbit, like the recent observation that coupling with spurious signals coming from the environment causes intermittent subharmonic windows. Based on this approach, we also propose a new controller that can significantly extend the parameter range for nominal period-1 operation.
\end{abstract}

Index Terms-Bifurcation, buck converter, differential inclusions, discontinuous systems, Filippov systems, power electronics.

\section{INTRODUCTION}

$\mathbf{P}$ OWER electronic circuits are switching dynamical systems characterized by discrete switching events that make the system toggle between two or more sets of differential equations. Because of this switching nature, the study of the stability of power electronic circuits requires special techniques. First, one has to obtain the stability of a periodic orbit rather than that of an equilibrium point for which most tools in control theory are developed. Second, the periodic orbit contains

Manuscript received January 4, 2006; revised March 7, 2007. This paper was recommended by Associate Editor M. D. Ilic.

D. Giaouris, B. Zahawi, and V. Pickert are with the School of Electrical, Electronic and Computer Engineering, University of Newcastle, Newcastle-uponTyne, NE1 7RU, U.K. (e-mail: Damian.Giaouris@ newcastle.ac.uk).

S. Banerjee is with the Centre for Theoretical Studies and Department of Electrical Engineering, Indian Institute of Technology, Kharagpur 721302, India (e-mail: soumitro@iitkgp.ac.in).

Digital Object Identifier 10.1109/TCSI.2008.916443 passage through more than one subsystem. One obviously has to locate the periodic orbit before the problem of its stability can be addressed. Traditionally, power electronics practitioners use the technique of averaging [1], [2] for obtaining some information about the stability and dynamic behavior of power electronic converters. However, it has been shown that, while the averaging method can capture the instabilities that occur on slow time scales, it effectively acts as a low-pass filter and ignores all phenomena that take place at clock frequency [3], [4]. It is known that the fast-scale instabilities that may develop in the voltage and current waveforms at clock frequency result in subharmonic and chaotic behavior [5]-[7]. It is therefore a matter of great importance to be able to analyze and predict such instabilities.

The method of sampled-data modeling was developed in the early 1980s and was systematically put forward by Verghese et al. in [8] and [9]. In the early 1990s, taking a clue from the method of Poincaré section used in the study of nonlinear dynamics, Deane and Hamill [10] put forward the equivalent concept of the iterated map as a model of power converters (for details, see [3] and [11]). In this method, the states of the converter are sampled in synchronism with the clock (called stroboscopic sampling) to obtain the discrete-time map in the form $\boldsymbol{x}_{n+1}=\boldsymbol{f}\left(\boldsymbol{x}_{n}\right)$. The iteration of the map represents the evolution of the state in discrete time, and the fixed point of the map represents the periodic orbit in continuous time. Once the nonlinear map is obtained, one can locally linearize it at the fixed point, and the eigenvalues of the Jacobian matrix determine the stability of the fixed point to small perturbations.

This method has been successful in analyzing the stability of periodic orbits especially in those systems where linearization of the map can be obtained in closed form. For example, in [12] and [13], the expressions for the nonlinear map in current-mode-controlled converters were obtained-whose local linearization at the fixed point reflected the stability of the orbit. However, in many other control schemes-notably in the common voltage-mode-controlled converters-the map cannot be obtained in closed form because of the transcendental form of the equations involved. In such systems, though it is possible to obtain the map numerically and thus it is possible to a obtain bifurcation diagram by iterating the map, studying the stability of specific periodic orbits poses a problem. Methods have been developed to study the stability of such systems, as outlined in Section II.

In an unconnected line of development occurring in the erstwhile Soviet Union, scientists like Aizerman, Gantmakher, 
and Filippov [14], [15] developed a mathematical formalism applicable to systems with a discontinuous right-hand side. The method was subsequently applied to mechanical systems undergoing stick-slip vibrations or impacting motion [16], [17], with very fruitful results. Since power electronic circuits also come under the general class of systems with discontinuous right-hand side, the natural question is: can Filippov's theory provide any new insight into analyzing the stability of power electronic circuits? In this paper, we probe this question. We apply Filippov's method of differential inclusions to an example power electronic circuit - the voltage-mode-controlled dc-dc buck converter-and develop a method to obtain the linearization around a periodic orbit. This is obtained as the fundamental solution matrix over a complete cycle (the so-called monodromy matrix), which is composed of the state transition matrices for the pieces of the orbit that lie in the individual subsystems, and the "saltation matrix"- the transition matrix that connects the perturbation just before a switching to that just after. The approach thus provides an alternative method of obtaining the Jacobian of the Poincaré map when the nonlinear map cannot be explicitly derived. In that sense, the Filippov approach achieves the same result as that obtained by some of the existing approaches outlined in Section II. However, the Filippov's method is more straightforward and, as we show in this paper, offers some additional insights into the behavior of the system. It may be noted that, though there have been reports of the application of Filippov's method in control theory literature [18]-[20], this paper contains the first application of this approach to power electronic circuits.

Using the example of a voltage-mode-controlled buck converter, we demonstrate how the monodromy matrix can be calculated for power electronic systems composed of linear subsystems. The eigenvalues of the monodromy matrix directly indicate the stability of the orbit to small perturbations. We show that this approach explains why a slight change of some of the system parameters may induce fast-scale instability even though the duty ratio changes only by an insignificant extent. We also illustrate how the method can be used in the design of power electronic converters, by identifying the parameter space region for nominal period-1 operation. We then provide numerical as well as experimental evidence to validate the theoretical results.

The power of the method developed in this paper is further illustrated by providing analytical explanation of two recent observations - that coupling with spurious sinusoidal signal causes the appearance of intermittent chaotic and subharmonic windows [21] and that a sinusoidally varying parametric perturbation can control the fast-scale instability [22]. Based on the above, we propose an improvement of the control scheme through the consideration of an optimum eigenvalue location combined with a supervising controller.

\section{Stability ANalysis of Periodic Orbits}

Here, we briefly review existing methods of stability analysis of limit cycles in power electronic circuits. Since most of the analytical tools used for this study stem from similar methods used for smooth systems, we first discuss such systems as a stepping stone for the analysis that will follow.

\section{A. Smooth Systems}

A system is said to be smooth if its mathematical model can be described by a set of differential equations

$$
\frac{\mathrm{d} \boldsymbol{x}(t)}{\mathrm{d} t}=\boldsymbol{f}(\boldsymbol{x}, t, \rho)
$$

where $f$ is differentiable everywhere in a given domain and $\rho$ is a system parameter. The stability analysis of a periodic orbit in such a system is based on the idea that, if a small perturbation is added and the solution converges back to the orbit, then the orbit is stable. There are three general approaches where this concept is used: 1) the trajectory sensitivity analysis; 2) the Poincaré map; and 3) the Floquet theory. All of these methods describe the stability of a limit cycle and hence there are many similarities between all three. In all cases, one needs to consider three factors: a local approximation, a solution of a matrix differential equation, and the use of a stability criterion.

1) Trajectory Sensitivity Approach: The method of trajectory sensitivity can generally be applied to any orbit which may not necessary be closed. The main concept here is to investigate how the solution (or trajectory) under study behaves in response to small changes in the initial conditions or other parameters [23].

Assuming a general nonautonomous system

$$
\dot{\boldsymbol{x}}=\boldsymbol{f}(\boldsymbol{x}, t), \quad \boldsymbol{x}\left(t_{0}\right)=\boldsymbol{x}_{0}
$$

the solution, according to the fundamental theorem of calculus, must satisfy

$$
\boldsymbol{\varphi}\left(t, t_{0}, \boldsymbol{x}_{0}\right)=\boldsymbol{x}_{0}+\int_{t_{0}}^{t} \boldsymbol{f}\left(\boldsymbol{\varphi}\left(\tau, t_{0}, \boldsymbol{x}_{0}\right), \tau\right) \mathrm{d} \tau
$$

By taking the partial derivative with respect to the initial condition, we obtain

$$
\frac{\partial \boldsymbol{\varphi}\left(t, t_{0}, \boldsymbol{x}_{0}\right)}{\partial \boldsymbol{x}_{0}}=\boldsymbol{I}+\int_{t_{0}}^{t} \mathbf{A}\left(\tau, \boldsymbol{x}_{0}\right) \frac{\partial \boldsymbol{\varphi}\left(\tau, t_{0}, \boldsymbol{x}_{0}\right)}{\partial \boldsymbol{x}_{0}} \mathrm{~d} \tau
$$

where

$$
\mathbf{A}\left(t, \boldsymbol{x}_{0}\right)=\frac{\partial \boldsymbol{f}\left(\boldsymbol{\varphi}\left(t, t_{0}, \boldsymbol{x}_{0}\right), t\right)}{\partial \boldsymbol{\varphi}\left(t, t_{0}, \boldsymbol{x}_{0}\right)} .
$$

By differentiating (4) with respect to time, we obtain

$$
\frac{\mathrm{d}}{\mathrm{d} t}\left(\frac{\partial \boldsymbol{\varphi}\left(t, t_{0}, \boldsymbol{x}_{0}\right)}{\partial \boldsymbol{x}_{0}}\right)=\mathbf{A}\left(t, \boldsymbol{x}_{0}\right) \frac{\partial \boldsymbol{\varphi}\left(t, t_{0}, \boldsymbol{x}_{0}\right)}{\partial \boldsymbol{x}_{0}} .
$$

The solution of the matrix differential equation (6) is called the sensitivity function [23] or trajectory sensitivity [24]. The sensitivity of the flow to initial condition can be obtained by the Taylor series expansion [24]

$$
\Delta \boldsymbol{\varphi}\left(t, t_{0}, \boldsymbol{x}_{0}\right)=\frac{\partial \boldsymbol{\varphi}\left(t, t_{0}, \boldsymbol{x}_{0}\right)}{\partial \boldsymbol{x}_{0}} \Delta \boldsymbol{\varphi}\left(t_{0}, t_{0}, \boldsymbol{x}_{0}\right) .
$$

2) Poincaré Map Approach: Though a much older technique, this is effectively a specific case of the trajectory sensitivity approach for periodic systems. In the Poincaré map approach, the continuous orbit is represented by a discrete map. In the case of 
a nonautonomous system, the state is observed every $T \mathrm{~s}$, where $T$ is the period of the forcing function or external clock. If we assume that $t=t_{0}$ is an observation instant, then the sampled data model is a nonlinear function that will map $\boldsymbol{x}_{0}$ to $\boldsymbol{x}_{t_{0}+T}$ as

$$
\boldsymbol{\varphi}\left(T+t_{0}, t_{0}, \boldsymbol{x}_{0}\right)=\boldsymbol{x}_{0}+\int_{t_{0}}^{t_{0}+T} \boldsymbol{f}\left(\boldsymbol{\varphi}\left(\tau, t_{0}, \boldsymbol{x}_{0}\right), \tau\right) d \tau
$$

The stability of this map can be deduced by taking the linear approximation (the Jacobian matrix) at its fixed point. Since the fixed point is $\boldsymbol{x}_{0}$, the linearized map is given by (4), where $t=$ $t_{0}+T$, i.e., the Jacobian matrix is the same as the trajectory sensitivity for $t=t_{0}+T$. Similarly, (6) has to be solved to analyze the stability. The behavior of the perturbations around the fixed point is given by (7), again for $t=t_{0}+T$.

3) Floquet Theory: With the above two methods, one studies how the perturbed trajectory behaves and the stability is then deduced by checking if the perturbations will increase or decrease. Another possible approach is to study the perturbations directly, i.e., if $\boldsymbol{x}_{p}(t)=\boldsymbol{\varphi}\left(t, t_{0}, \boldsymbol{x}_{0}\right)$ is the original periodic orbit and $\boldsymbol{x}_{\bar{p}}(t)$ is the perturbed trajectory, then the perturbation is $\Delta \boldsymbol{x}(t)=\boldsymbol{x}_{\bar{p}}(t)-\boldsymbol{x}_{p}(t)$. By using conventional linearization methods (Taylor series) around the periodic orbit $\boldsymbol{x}_{\boldsymbol{p}}(t)$, it can easily be shown that the perturbation $\Delta \boldsymbol{x}(t)$ can be modeled by a homogeneous linear time-varying model [17]

$$
\frac{\mathrm{d} \Delta \boldsymbol{x}(t)}{\mathrm{d} t}=\left.\frac{\partial \boldsymbol{f}(\boldsymbol{x}, t)}{\partial \boldsymbol{x}}\right|_{\boldsymbol{x}_{=\boldsymbol{x}_{p}}} \Delta \boldsymbol{x}(t)=\mathbf{A}\left(t, \boldsymbol{x}_{p}\right) \Delta \boldsymbol{x}(t) .
$$

Unfortunately, the eigenvalues of the state matrix cannot be used to determine the stability as it is time varying. This problem may be overcome by using the fundamental solution matrix, which is given by

$$
\boldsymbol{\Phi}\left(t, t_{0}, \Delta \boldsymbol{x}_{0}\right)=\left[\boldsymbol{\varphi}_{1}\left(t, t_{0}, \Delta \boldsymbol{x}_{0}\right), \boldsymbol{\varphi}_{2}\left(t, t_{0}, \Delta \boldsymbol{x}_{0}\right), \ldots\right]
$$

where $\varphi_{i}\left(t, t_{0}, \Delta x_{0}\right)$ are linear independent solutions of the system with the property that any other solution can be written as a linear combination of $\varphi_{i}\left(t, t_{0}, \Delta x_{0}\right)$. The state transition matrix is the fundamental solution matrix satisfying the condition $\boldsymbol{\Phi}\left(t_{0}, t_{0}, \Delta \boldsymbol{x}_{0}\right)=\boldsymbol{I}$. The state transition matrix can be defined for any homogeneous system, and, in the case where the state matrix is time-invariant, it can easily be calculated by using the matrix exponential $\boldsymbol{\Phi}\left(t, t_{0}\right)=e^{\mathbf{A}\left(t-t_{0}\right)}$. However, since $\mathbf{A}\left(t, \boldsymbol{x}_{p}\right)$ is time varying, no closed-form expression exists for the state transition matrix, and we must solve the matrix differential equation

$$
\frac{\mathrm{d} \boldsymbol{\Phi}\left(t, t_{0}, \Delta \boldsymbol{x}_{0}\right)}{\mathrm{d} t}=\mathbf{A}\left(t, \boldsymbol{x}_{p}\right) \boldsymbol{\Phi}\left(t, t_{0}, \Delta \boldsymbol{x}_{0}\right) .
$$

If the solution of (11) (i.e., the state transition matrix) is evaluated for $t=T+t_{0}$, then the resulting matrix is called the monodromy matrix. One basic property of the monodromy matrix [17] is that

$$
\Delta \boldsymbol{x}(k T)=\boldsymbol{\Phi}^{k}\left(T+t_{0}, t_{0}, \Delta \boldsymbol{x}_{0}\right) \Delta \boldsymbol{x}_{0}
$$

This implies that, if the absolute value of the eigenvalues (also called Floquet multipliers) of the monodromy matrix are all less than 1 , then the perturbation $\Delta \boldsymbol{x}$ will converge to zero and thus the system is stable.

Comparing (6) and (11), it is interesting to note that the monodromy matrix is effectively the trajectory sensitivity at $t=$ $t_{0}+T$, which in turn is the Jacobian of the Poincare map [24], [25]. Thus, all three approaches finally yield the same result.

\section{B. Nonsmooth Systems}

A system is said to be nonsmooth or piecewise smooth if it is described by equations of the form

$$
\dot{\boldsymbol{x}}=f(\boldsymbol{x}, t, \rho)= \begin{cases}f_{1}(\boldsymbol{x}, t, \rho), & \text { for } \boldsymbol{x} \in R_{1} \\ f_{2}(\boldsymbol{x}, t, \rho), & \text { for } \boldsymbol{x} \in R_{2} \\ \vdots & \\ f_{n}(\boldsymbol{x}, t, \rho), & \text { for } \boldsymbol{x} \in R_{n}\end{cases}
$$

where $R_{1}, R_{2}$, etc., are different regions of the state space, separated by $(n-1)$-dimensional surfaces given by algebraic equations of the form $\Gamma_{n}(\boldsymbol{x})=0$, called "switching manifolds." Application of the above methods to nonsmooth systems demands some special treatment, because the original and perturbed solutions may not undergo switching at the same time. Thus, in the neighborhood of a switching event, the original periodic solution and the perturbed trajectory are not given by the same vector field.

1) Trajectory Sensitivity: Hiskens and Pai [26] addressed the problem of defining (5) in such systems with reference to a general discrete-algebraic-differential (DAD) model. Here, we will briefly describe this method when there is one switching per cycle, as it is closely related to the method proposed by Filippov. The DAD model can be given by

$$
\begin{array}{rlr}
\dot{\boldsymbol{x}}(t) & =\boldsymbol{f}(\boldsymbol{x}, \boldsymbol{y}) \\
s(\boldsymbol{x}, \boldsymbol{y}) & =0 \\
g^{-}(\boldsymbol{x}, \boldsymbol{y}) & =0, \quad s(\boldsymbol{x}, \boldsymbol{y})<0 \\
g^{+}(\boldsymbol{x}, \boldsymbol{y}) & =0, \quad s(\boldsymbol{x}, \boldsymbol{y})>0 \\
\boldsymbol{x}^{+} & =\mathbf{D}(\boldsymbol{x}, \boldsymbol{y})
\end{array}
$$

where $f$ defines the vector field, $s$ is a scalar equation that describes the switching surface, $g^{-}$is a scalar equation that is satisfied before the switching, $g^{+}$is a scalar equation that is satisfied after the switching, $\boldsymbol{x}$ 's are the dynamical states of the system (e.g., inductor current and capacitor voltage), $\boldsymbol{y}$ 's are other algebraic variables (like supply and reference voltages), and $\mathbf{D}$ is a vector function that describes how the state will jump just after the switching. Hiskens et al. used this model to find the required jump map. Very briefly, just before the switching, the two conditions that must be satisfied are

$$
\begin{aligned}
& s\left(\boldsymbol{x}^{-}, \boldsymbol{y}^{-}\right)=0 \\
& g^{-}\left(\boldsymbol{x}^{-}, \boldsymbol{y}\right)^{-}=0
\end{aligned}
$$

where $\boldsymbol{x}^{-}$and $\boldsymbol{y}^{-}$are the state and algebraic variables immediately before the switching. Furthermore, by using the chain rule, the derivative of the state vector just before the switching is given by

$$
\frac{\mathrm{d} \boldsymbol{x}\left(\tau^{-}\right)}{\mathrm{d} \boldsymbol{x}_{0}}=\left.\left(\frac{\partial \boldsymbol{x}}{\partial \boldsymbol{x}_{0}}+\frac{\partial \boldsymbol{x}}{\partial \tau} \frac{\mathrm{d} \tau}{\mathrm{d} \boldsymbol{x}_{0}}\right)\right|_{t=\tau^{-}}
$$


where $\tau^{-}$is the instant just before the switching. Also

$$
\left.\frac{\mathrm{d} \boldsymbol{x}}{\mathrm{d} t}\right|_{t=\tau^{-}}=\boldsymbol{f}\left(\boldsymbol{x}^{-}, \boldsymbol{y}^{-}\right)=\boldsymbol{f}^{-} .
$$

By using a Taylor series on $s$ and $g$ just before the switching, we obtain

$$
\begin{array}{r}
s_{x} \mathrm{~d} \boldsymbol{x}^{-}+s_{y} \mathrm{~d} \boldsymbol{y}^{-}=0 \\
g_{x}^{-} \mathrm{d} \boldsymbol{x}^{-}+g_{y}^{-} \mathrm{d} \boldsymbol{y}^{-}=0
\end{array}
$$

where $s_{x}, g_{x}, s_{y}$, and $g_{y}$ are partial derivatives with respect to $\boldsymbol{x}$ and $\boldsymbol{y}$, respectively. By combining (15)-(17), we can find an expression for $\mathrm{d} \tau / \mathrm{d} \boldsymbol{x}_{0}$ which will describe how the switching time changes with small changes in initial conditions. This can then be used again in (15) to define how the solution will alter by small changes in initial conditions. A similar approach can be applied for the solution after the trajectory. $\mathbf{D}$ can then be used to relate these two and hence define the jump matrix that describes the relation between $\mathrm{d} \boldsymbol{x}\left(\tau^{-}\right) / \mathrm{d} \boldsymbol{x}_{0}$ and $\mathrm{d} \boldsymbol{x}\left(\tau^{+}\right) / \mathrm{d} \boldsymbol{x}_{0}$. If there is no discontinuity (i.e., $\boldsymbol{x}^{-}=\boldsymbol{x}^{+}$), by using the linear approximation given in (7) for both sides of the switching, the map reduces to a simple form similar to the saltation matrix [14] and part of the Jacobian of the Poincaré map [27], [28].

2) Poincaré Map: For power electronic circuits, the sampled-data model developed by Verghese $e t$ al. (see [8] for an account of its history) and the equivalent Poincaré map approach proposed by Deane and Hamill [10] essentially samples the state variables discretely at the clock instants if the system is nonautonomous (like $\mathrm{dc}-\mathrm{dc}$ converters under voltage or current mode control) or at the points of intersection of the trajectory with a Poincaré surface in case of autonomous systems (like dc-dc converters under hysteresis control).

The sampled-data model is obtained as follows. In this exposition, we assume the system to be nonautonomous, with stroboscopic sampling in synchronism with the clock of period $T$. It is a reasonable assumption in $\mathrm{dc}-\mathrm{dc}$ converters that the subsystems are linear time-invariant (LTI), and the evolution in each subsystem is defined by a differential equation of the form

$$
\frac{\mathrm{d} \boldsymbol{x}(t)}{\mathrm{d} t}=\mathbf{A}_{i} \boldsymbol{x}(t)+\mathbf{B}_{i} \boldsymbol{u}(t)
$$

for $i=1,2, \ldots$. Assuming operation in the nominal period-1 steady state, in which there is only one switching in a clock cycle occurring at the time instant $d T$ where $d$ is the duty ratio, we can derive the following system equations.

Before the first switching in a clock cycle, the state evolves as

$$
\begin{aligned}
\boldsymbol{x}(d T) & =e^{\mathbf{A}_{1} d T} \boldsymbol{x}(0)+\int_{0}^{d T} e^{\mathbf{A}_{1}(d T-\tau)} \mathbf{B}_{1} u(\tau) \mathrm{d} \tau \\
& =\boldsymbol{\Phi}_{1}(d T, 0) \boldsymbol{x}(0)+\mathbf{I}_{1}(d) .
\end{aligned}
$$

After the switching and until the end of the clock cycle, the state evolves as

$$
\begin{aligned}
\boldsymbol{x}(T) & =e^{\mathbf{A}_{2}(T-d T)} \boldsymbol{x}(d T)+\int_{d T}^{T} e^{\mathbf{A}_{2}(T-\tau)} \mathbf{B}_{2} u(\tau) \mathrm{d} \tau \\
& =\boldsymbol{\Phi}_{2}(T, d T) \boldsymbol{x}(d T)+\mathbf{I}_{2}(d) .
\end{aligned}
$$

Assuming that there is no discontinuity in the state, one can take the final state before a switching instant to be equal to the initial state after the switching. This gives the sampled-data model of the system

$$
\begin{aligned}
\boldsymbol{x}(T) & =\boldsymbol{f}\left(\boldsymbol{x}_{0}, d\right) \\
& =\boldsymbol{\Phi}_{2}(T, d T)\left\{\boldsymbol{\Phi}_{1}(d T, 0) \boldsymbol{x}(0)+\mathbf{I}_{1}(d)\right\}+\mathbf{I}_{2}(d) .(18)
\end{aligned}
$$

For a periodic orbit, $\mathbf{x}(T)=\mathbf{x}(0)$, this gives

$$
\begin{aligned}
\boldsymbol{x}(0)=\left[\boldsymbol{I}-\boldsymbol{\Phi}_{2}(T, d T) \boldsymbol{\Phi}_{1}(d T, 0)\right]^{-1} & \\
& \times\left[\boldsymbol{\Phi}_{2}(T, d T) \mathbf{I}_{1}(d)+\mathbf{I}_{2}(d)\right] .
\end{aligned}
$$

The switching events occur when the algebraic equation

$$
h(\boldsymbol{x}(0), d)=0
$$

is satisfied. Substituting (19) into this equation, we have an equation involving only one unknown: the duty ratio $d$. This can be solved easily using any numerical routine. This procedure yields the location of the periodic orbit.

In order to study the stability of the limit cycle, we need to obtain the linearization of the sampled data model given by (18). Differentiating this with respect to $\boldsymbol{x}(0)$ and using the chain rule, we obtain

$$
\frac{\partial \boldsymbol{x}(T)}{\partial \boldsymbol{x}(0)}=\frac{\partial \boldsymbol{f}\left(\boldsymbol{x}_{0}, d\right)}{\partial \boldsymbol{x}(0)}+\frac{\partial \boldsymbol{f}\left(\boldsymbol{x}_{0}, d\right)}{\partial d} \frac{\partial d}{\partial \boldsymbol{x}(0)}
$$

which effectively is a special case of (4). Note that the duty ratio $d$ is a function of $x(0)$.

By a similar procedure, the switching condition (20) yields

$$
\frac{\partial h}{\partial \boldsymbol{x}(0)}+\frac{\partial h}{\partial d} \frac{\partial d}{\partial \boldsymbol{x}(0)}=0
$$

where $h=h(\boldsymbol{x}(0), d)$. By rearranging the last equation, we have

$$
\frac{\partial d}{\partial \boldsymbol{x}(0)}=-\left(\frac{\partial h}{\partial d}\right)^{-1} \frac{\partial h}{\partial \boldsymbol{x}(0)} .
$$

Substituting this into (21), we get

$$
\frac{\partial \boldsymbol{x}(T)}{\partial \boldsymbol{x}(0)}=\frac{\partial \boldsymbol{f}\left(\boldsymbol{x}_{0}, d\right)}{\partial \boldsymbol{x}(0)}-\frac{\partial \boldsymbol{f}\left(\boldsymbol{x}_{0}, d\right)}{\partial d}\left(\frac{\partial h}{\partial d}\right)^{-1} \frac{\partial h}{\partial \boldsymbol{x}(0)} .
$$

This gives the expression for the Jacobian matrix, which can then be evaluated depending on the specific converter topology. For example, Fang and Abed [3], [27], [28] showed that, for a voltage-mode-controlled converter, this yields the form given by the equation shown at the bottom of the next page. A different approach was used in [29] where an auxiliary state vector was used to simplify the analysis of the aforementioned Jacobian matrix for a resonant buck converter.

3) Floquet Theory: In order to use the Floquet theory in switching systems, it is necessary to take into account the change in the vector field at a switching event. This is the main contribution of this paper and will therefore be presented in the some detail in Sections III-VIII.

Before that, however, we would like to mention another method developed at the University of Tokushima, Japan [30], which locates the fixed point of the Poincaré map by the Newton-Raphson method, and in the process of convergence, obtains the relevant partial derivatives from which the Jacobian of the Poincare map can be derived. It thus obtains the fixed 


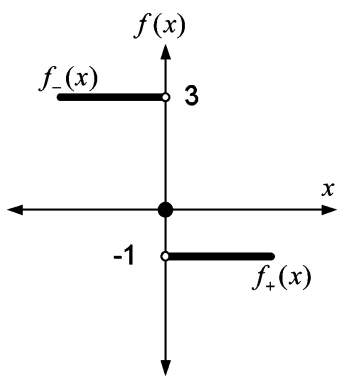

Fig. 1. Discontinuous system of Filippov type.

point and its Jacobian by the same numerical procedure. The technique is particularly useful in systems where the subsystems are nonlinear.

\section{OUTLINE OF FILIPPOV's MethoD}

Filippov showed that, in systems with a discontinuous righthand side, there can be ambiguity in the definition of solutions. To illustrate, consider the simple one-dimensional (1-D) system

$$
\dot{x}(t)= \begin{cases}f_{-}(x(t))=3, & \text { if } x(t)<0 \\ f_{0}(x(t))=1, & \text { if } x(t)=0 \\ f_{+}(x(t))=-1, & \text { if } x(t)>0\end{cases}
$$

shown in Fig. 1. If $x(0) \neq 0$, it has the solution

$$
x(t)= \begin{cases}3 t+C_{1}, & \text { if } x(t)<0 \\ -t+C_{2}, & \text { if } x(t)>0\end{cases}
$$

which reaches $x=0$ in finite time and stays there forever. This implies that $x=0$ and $\dot{x}=0$ but, according to (23) (when $x=0), \dot{x}=1 \neq 0$. Therefore, (23) cannot have a solution in the classical sense as (1) does.

To avoid this problem, Filippov suggested that $f_{0}(x(t))$ will not be a single valued function but a set valued function (as shown in Fig. 2) whose limits are the values of $f(x(t))$ before and after the switching

$$
\begin{aligned}
f_{0}(x(t)) & =\left[\min \left(f_{+}(x), f_{-}(x)\right), \max \left(f_{+}(x), f_{-}(x)\right)\right] \\
& =\left\{q f_{-}(x)+(1-q) f_{+}(x)\right\} \quad \forall q \in[0,1]
\end{aligned}
$$

which is the closed convex set containing $f_{-}(x(t))$ and $f_{+}(x(t))$, often denoted as $f_{0}(x(t))=$ $\overline{c o}\left\{f_{-}(x(t)), f_{+}(x(t))\right\}$. Hence, (23) can be written as

$$
\dot{x}(t) \in F(x(t))= \begin{cases}f_{-}(x(t)), & \text { if } x(t)<0 \\ \overline{c o}\left\{f_{-}(x(t)), f_{+}(x(t))\right\}, & \text { if } x(t)=0 \\ f_{+}(x(t)), & \text { if } x(t)>0 .\end{cases}
$$

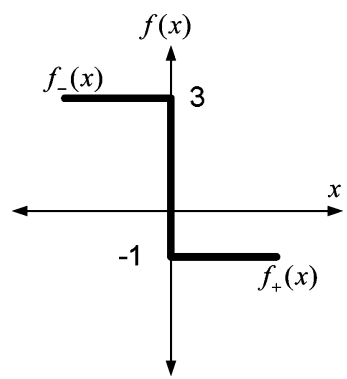

Fig. 2. Set valued function.

The extension of (23) into (24) is known as Filippov's convex method and the solution of (24) is referred to as Filippov solution [17]. The existence of a Filippov solution is guaranteed if $F$ is upper semi-continuous (the extension of continuity into set valued functions). Uniqueness of a Filippov solution for every initial condition can be guaranteed if the orbit spends almost zero time on the switching manifold, i.e., if there is a transversal intersection of the orbit with the switching manifold.

In studying the stability of periodic orbits, one has to continuously evaluate the Jacobian that appears in (9) which describes the evolution of the perturbations. As seen earlier, at the switching this breaks down as the perturbed solution will reach the switching after (or before) the original periodic orbit. The Filippov approach considers small perturbations to the initial condition, and studies how the perturbations evolve as the continuous-time trajectory traverses the complete clock period $T$. As in the trajectory sensitivity approach, we need a map that will relate the perturbation vectors before and after a switching, i.e., from a point where both the periodic orbit and the perturbed orbit have not crossed the switching manifold to a point where both have crossed. It has to be noted that the concept of using maps to relate the perturbations before and after the switching has also been applied in discontinuous mechanical systems [31], [32]. This map $\boldsymbol{S}$, referred to as the saltation matrix [17], is given by

$$
\Delta \boldsymbol{x}\left(t_{\Sigma}^{+}\right)=\boldsymbol{S} \Delta \boldsymbol{x}\left(t_{\Sigma}^{-}\right) .
$$

Suppose an orbit is passing from subsystem- 1 given by the vector field $f_{-}(x(t))$ to subsystem-2 given by the vector field $\boldsymbol{f}_{+}(\boldsymbol{x}(t))$ at a switching manifold $\Sigma$ defined by the algebraic equation $h(\boldsymbol{x}(t), t)=0$. It has been shown [15], [17] that, when there is a transversal intersection, the saltation matrix is given by

$$
\boldsymbol{S}=\boldsymbol{I}+\frac{\left(\boldsymbol{f}_{-}-\boldsymbol{f}_{+}\right) \boldsymbol{n}^{T}}{\boldsymbol{n}^{T} \boldsymbol{f}_{+}+\left.\frac{\partial h}{\partial t}\right|_{t=t_{\Sigma}}}
$$

$$
e^{\mathbf{A}_{2}(T-d T)}\left(\mathbf{I}-\frac{\left(\left(\mathbf{A}_{1}-\mathbf{A}_{2}\right) \boldsymbol{x}(d T)+\left(\mathbf{B}_{1}-\mathbf{B}_{2}\right) \mathbf{u}\right)\left[\begin{array}{ll}
1 & 0
\end{array}\right]}{\left[\begin{array}{ll}
1 & 0
\end{array}\right]\left(\mathbf{A}_{1} \boldsymbol{x}(d T)+\mathbf{B}_{1} \mathbf{u}\right)-\left.\frac{\mathrm{d} h}{\mathrm{~d} t}\right|_{t=d T}}\right) e^{\mathbf{A}_{1} d T}
$$


where $t_{\Sigma}$ is the time at which the orbit crosses the manifold, and $\boldsymbol{n}$ is a vector normal to the switching surface. The vector field evaluated on one side of the switching manifold, i.e., $\lim _{t \uparrow t_{\Sigma}}\left(f_{-}(\boldsymbol{x}(t))\right.$, is abbreviated as $\boldsymbol{f}_{-}$, and that in the other side, i.e., $\lim _{t \downarrow t_{\Sigma}}\left(\boldsymbol{f}_{+}(\boldsymbol{x}(t))\right.$, is abbreviated as $\boldsymbol{f}_{+}$. The form of the saltation matrix is derived using a local approximation of the perturbed and original solutions, and the scalar function that defines the switching manifold. In that sense the procedure is similar to that in the trajectory sensitivity approach using the DAD model, but now the scalar function of algebraic variables $(g)$ is included in the scalar function $h$ that defines the location the switching manifold.

Suppose a periodic orbit starts at $t_{0}$ in subsystem-1, intersects the switching manifold at $t_{\Sigma}$ and goes over to subsystem-2, and finally the periodic orbit closes at $t=T$. Then, $\boldsymbol{\Phi}\left(t_{0}+T, t_{0}, \boldsymbol{x}\left(t_{0}\right)\right)$, is the monodromy matrix for the piecewise system. Once the transition matrices in each subsystem and the saltation matrix corresponding to the switching are obtained, one can compose the monodromy matrix as

$$
\begin{aligned}
\boldsymbol{\Phi}\left(t_{0}+\right. & \left.T, t_{0}, \boldsymbol{x}\left(t_{0}\right)\right) \\
& =\boldsymbol{\Phi}\left(t_{0}+T, t_{\Sigma^{+}}, \boldsymbol{x}\left(t_{\Sigma^{+}}\right)\right) \cdot \boldsymbol{S} \cdot \boldsymbol{\Phi}\left(t_{\Sigma^{-}}, t_{0}, \boldsymbol{x}\left(t_{0}\right)\right)
\end{aligned}
$$

where $t_{\Sigma^{-}}$is the time instant just before crossing the switching manifold and $t_{\Sigma^{+}}$is that just after crossing it. If each subsystem is linear time invariant, the state transition matrix for the travel through each system can be obtained using the matrix exponential.

Note that the form of the monodromy matrix is sufficiently general. If the orbit contains passage through more number of subsystems with a number of crossings (as, for example, in a high-period orbit), one just has to compose the monodromy matrix out of the state transition matrices for the passage through each subsystem, and the saltation matrices related to the crossings. This will greatly simplify the analysis as each crossing can be treated separately. Furthermore, the Filippov approach can be used to study systems that exhibit sliding modes [17], and hence it can be applied to discontinuous conduction modes also.

The monodromy matrix essentially represents the linearized system integrated around a periodic orbit, and hence its eigenvalues represent the Floquet multipliers. If they lie within the unit circle, the orbit is stable. The Filippov method thus gives the same result as the other methods outlined in Section II, though by a different route. Note that for the nominal period-1 operation of the buck converter, Fang and Abed [27], [28] derived the same form by differentiating the Poincaré map (see the expression at the end of Section II).

\section{Buck CONVERTER AND ITS MATHEMATICAL MODEL}

The voltage-mode-controlled buck converter circuit shown in Fig. 3 is a piecewise affine system described by

$$
\begin{aligned}
\frac{\mathrm{d} i(t)}{\mathrm{d} t} & = \begin{cases}\frac{v_{\text {in }}-v(t)}{L}, & S \text { is conducting } \\
-\frac{v(t)}{L}, & S \text { is blocking }\end{cases} \\
\frac{\mathrm{d} v(t)}{\mathrm{d} t} & =\frac{i(t)-\frac{v(t)}{R}}{C} .
\end{aligned}
$$

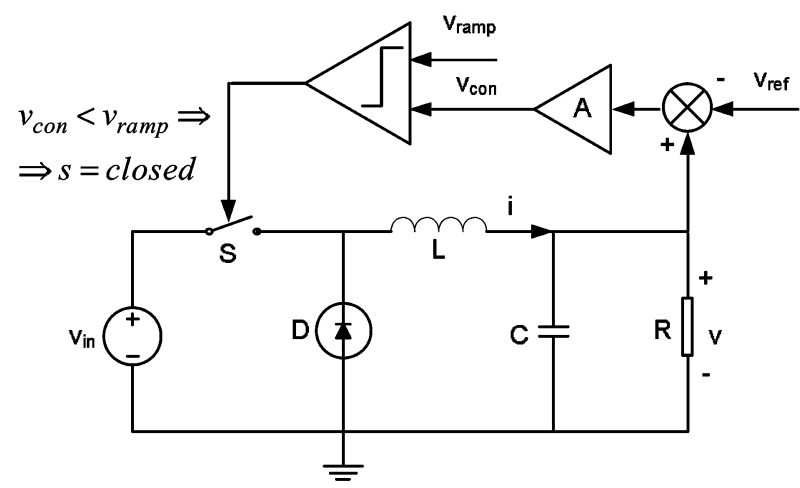

Fig. 3. Voltage-mode-controlled buck dc-dc converter.

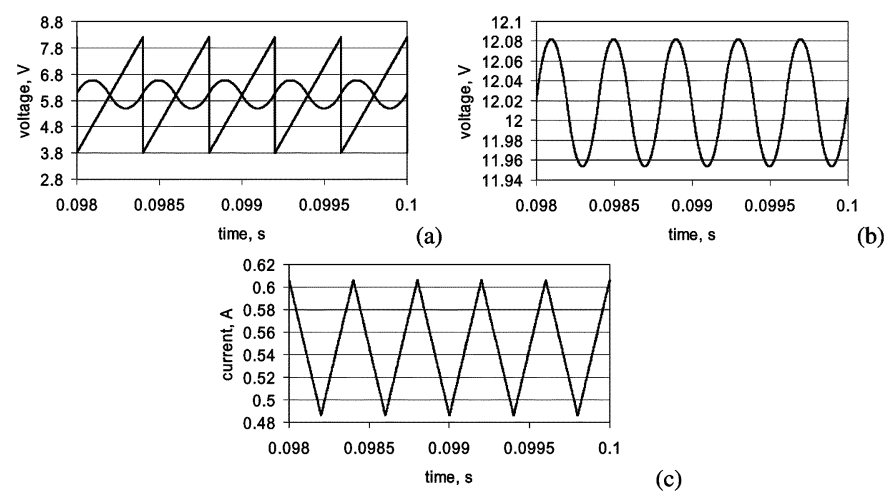

Fig. 4. Nominal period-1 operation of the buck converter. (a) Control and ramp signals. (b) Output voltage. (c) Output current. The parameter values are $V_{\text {in }}=$ $24 \mathrm{~V}, V_{\text {ref }}=11.3 \mathrm{~V}, L=20 \mathrm{mH}, R=22 \Omega, C=47 \mu \mathrm{F}, A=8.4$, $T=1 / 2500 \mathrm{~s}$, and the ramp signal varies from 3.8 to $8.2 \mathrm{~V}$.

It is obvious from (28) and (29) that there is a discontinuity in the right-hand side when the main switching element $S$ passes from conduction to blocking mode and vice versa.

Generally, there is some closed-loop scheme to control the switching so that the output voltage is kept constant. In this paper, we consider the voltage-mode control in which the output voltage is compared with a reference voltage to generate a control voltage signal $v_{\text {con }}=A\left(v(t)-V_{\text {ref }}\right)$, where $A$ is the gain of the feedback loop. ${ }^{1}$ The error voltage is then compared with a periodic sawtooth signal $v_{\text {ramp }}$ to generate the switching signal as follows:

if $v_{\text {con }}<v_{\text {ramp }}(t), S$ is turned on;

if $v_{\text {con }}>v_{\text {ramp }}(t), S$ is turned off.

Under normal conditions, the output of the converter will be a dc voltage with a periodic ripple, with a mean value close to the desired voltage and a period that is equal to the period of the PWM ramp signal (referred to as period-1 waveform), as shown in Fig. 4. In Fig. 5, the same orbit is shown in the $v-i$ state space, with nine points on the orbit marked. The beginning of the ramp period coincides with the time-point marked 1 . As the orbit progresses in time, the ramp voltage sweeps from left to right, and, at time point 5, they intersect. The switch turns on, and

\footnotetext{
${ }^{1}$ In many applications, a PI controller is used. However, in this paper, we consider a proportional controller for two reasons. First, the additional capacitor in the feedback loop increases the system dimension to three. Our focus here is to develop a new method for analyzing stability — not to study a particular highdimensional system. Second, we have deliberately chosen an example system that has been studied by others [6], [7], [10], [27] so that the reader can easily cross-examine the results.
} 


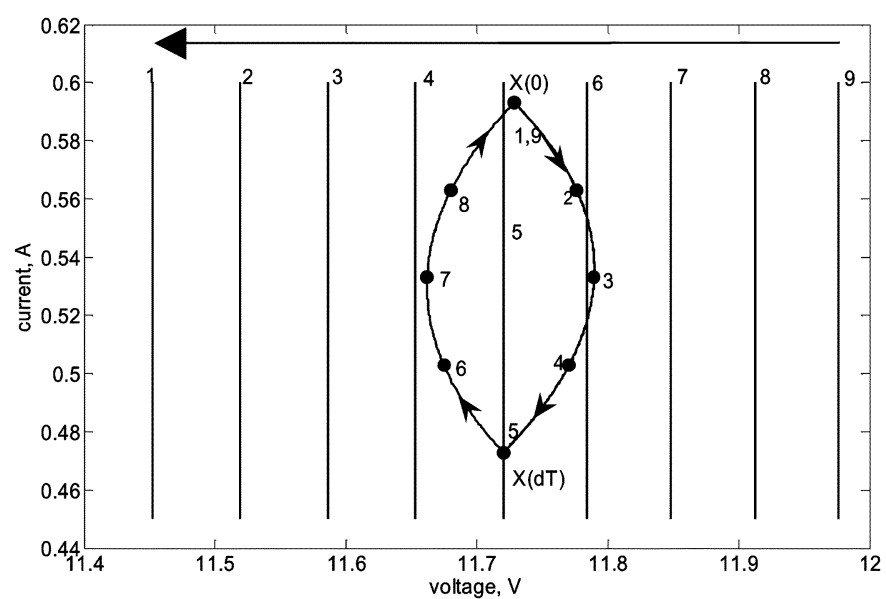

Fig. 5. Period-1 limit cycle: the nine different time points on that orbit and the location of the time-varying switching surface at these instants. After the ninth point, the surface goes back to 1 , repeating the periodic cycle.

remains in that state till the end of the ramp period (time point 9). The orbit is periodic if the state at time point 9 coincides with that at time point 1 . It has been shown that as a parameter (say, the input voltage) is varied, the orbit may lose stablity and highperiodic or chaotic orbits may occur [6], [7], [10]. Our concern here is to determine the stability of this orbit.

\section{Application OF THE FilipPOV APPROACH TO THE BUCK CONVERTER}

By defining $x_{1}(t)=v(t)$ and $x_{2}(t)=i(t),(28)$ and (29) may be written as

$$
\dot{\boldsymbol{x}}= \begin{cases}\mathbf{A}_{s} \boldsymbol{x}+\mathbf{B u}, & A\left(x_{1}(t)-V_{\text {ref }}\right)<v_{\text {ramp }}(t) \\ \mathbf{A}_{s} \boldsymbol{x}, & A\left(x_{1}(t)-V_{\text {ref }}\right)>v_{\text {ramp }}(t) .\end{cases}
$$

where

$$
\mathbf{A}_{s}=\left[\begin{array}{cc}
\frac{-1}{R C} & \frac{1}{C} \\
\frac{-1}{L} & 0
\end{array}\right] \quad \mathbf{B u}=\left[\begin{array}{c}
0 \\
\frac{1}{L}
\end{array}\right] V_{\mathrm{in}} .
$$

The switching hypersurface $(h)$ is given by

$$
\begin{aligned}
& h(\boldsymbol{x}(t), t)=x_{1}(t)-V_{\text {ref }}-\frac{v_{\text {ramp }}(t)}{A}=0, \quad A \neq 0 . \\
& s v_{\text {ramp }}(t)=V_{L}+\left(V_{U}-V_{L}\right)\left(\frac{t}{T} \bmod 1\right) .
\end{aligned}
$$

The two-dimensional state space is divided into three parts

$$
P_{-} \cup \Sigma \cup P_{+}=\mathbb{R}^{2}
$$

where

$$
\begin{aligned}
P_{-} & =\left\{\boldsymbol{x} \in \mathbb{R}^{2}: h(\boldsymbol{x}(t), t)<0\right\} \\
P_{+} & =\left\{\boldsymbol{x} \in \mathbb{R}^{2}: h(\boldsymbol{x}(t), t)>0\right\} \\
\Sigma & =\left\{\boldsymbol{x} \in \mathbb{R}^{2}: h(\boldsymbol{x}(t), t)=0\right\} .
\end{aligned}
$$

Equation (30) can be written as an upper semi-continuous Filippov inclusion

$$
\dot{\boldsymbol{x}} \in \boldsymbol{F}(\boldsymbol{x}(t))= \begin{cases}\boldsymbol{f}_{-}(\boldsymbol{x}(t)), & \text { if } \boldsymbol{x},(t) \in P_{-} \\ \overline{c o}\left\{\boldsymbol{f}_{-}(\boldsymbol{x}(t)), \boldsymbol{f}_{+}(\boldsymbol{x}(t))\right\}, & \text { if } \boldsymbol{x},(t) \in \Sigma \\ \boldsymbol{f}_{+}(\boldsymbol{x}(t)), & \text { if } \boldsymbol{x},(t) \in P_{+}\end{cases}
$$

where

$$
\begin{aligned}
& \boldsymbol{f}_{-}(\boldsymbol{x}(t))=\left[\begin{array}{c}
\frac{x_{2}(t)}{C}-\frac{x_{1}(t)}{R C} \\
\frac{\left(V_{\text {in }}-x_{1}(t)\right)}{L}
\end{array}\right] \\
& \boldsymbol{f}_{+}(\boldsymbol{x}(t))=\left[\begin{array}{c}
\frac{x_{2}(t)}{C}-\frac{x_{1}(t)}{R C} \\
-\frac{x_{1}(t)}{L}
\end{array}\right] .
\end{aligned}
$$

At the two sides of the switching hypersurface $f_{-}(x(t)) \neq$ $\boldsymbol{f}_{+}(\boldsymbol{x}(t))$ and, therefore, the system has discontinuous vector field. The convex hull is defined as

$$
\begin{aligned}
& \overline{c o}\left\{\boldsymbol{f}_{-}(\boldsymbol{x}(t)), \boldsymbol{f}_{+}(\boldsymbol{x}(t))\right\} \\
& =\left[\begin{array}{c}
\frac{x_{2}(t)}{C}-\frac{x_{1}(t)}{R C} \\
\overline{c o}\left\{\frac{V_{\text {in }}-x_{1}(t)}{L},-\frac{x_{1}(t)}{L}\right\}
\end{array}\right] \\
& =\left[\begin{array}{c}
\frac{x_{2}(t)}{C}-\frac{x_{1}(t)}{R C} \\
(1-q) \frac{V_{\text {in }}-x_{1}(t)}{L}-q \frac{x_{1}(t)}{L}
\end{array}\right] \quad \forall q \in[0,1] .
\end{aligned}
$$

The normal to the hypersurface is

$$
\boldsymbol{n}=\nabla h(\boldsymbol{x}(t), t)=\left[\begin{array}{c}
\frac{\partial h(\boldsymbol{x}(t), t)}{\partial x_{1}(t)} \\
\frac{\partial h(\boldsymbol{x}(t), t)}{\partial x_{2}(t)}
\end{array}\right]=\left[\begin{array}{l}
1 \\
0
\end{array}\right]
$$

Now, if we consider the period-1 orbit as shown in Figs. 4 and 5 , the solution intersects the switching manifold transversally and the orbit spends infinitely small time on the switching manifold. Therefore, Filippov's method of obtaining the saltation matrix and the monodromy matrix can be applied. ${ }^{2}$

The components of the saltation matrix (26) are obtained as

$$
\begin{aligned}
& \lim _{t \downarrow t_{\Sigma}} \boldsymbol{f}_{-}(\boldsymbol{x}(t))=\left[\begin{array}{c}
\frac{x_{2}\left(t_{\Sigma}\right)}{C}-\frac{x_{1}\left(t_{\Sigma}\right)}{R C} \\
\frac{\left(V_{\text {in }}-x_{1}\left(t_{\Sigma}\right)\right)}{L}
\end{array}\right] \\
& \lim _{t \uparrow t_{\Sigma}} \boldsymbol{f}_{+}(\boldsymbol{x}(t))=\left[\begin{array}{c}
\frac{x_{2}\left(t_{\Sigma}\right)}{C}-\frac{x_{1}\left(t_{\Sigma}\right)}{R C} \\
\frac{-x_{1}\left(t_{\Sigma}\right)}{L}
\end{array}\right]
\end{aligned}
$$

where $t_{\Sigma}$ is the switching instant.

Thus

$$
\begin{aligned}
\lim _{t \downarrow t_{\Sigma}} \boldsymbol{f}_{-}(\boldsymbol{x}(t))-\lim _{\boldsymbol{t} \uparrow t_{\Sigma}} \boldsymbol{f}_{+}(\boldsymbol{x}(t)) & =\left[\begin{array}{c}
0 \\
\frac{V_{\mathrm{in}}}{L}
\end{array}\right] \\
\left(\lim _{t \downarrow t_{\Sigma}} \boldsymbol{f}_{-}(\boldsymbol{x}(t))-\lim _{t \uparrow t_{\Sigma}} \boldsymbol{f}_{+}(\boldsymbol{x}(t)) \boldsymbol{n}^{T}\right. & =\left[\begin{array}{cc}
0 & 0 \\
\frac{V_{\mathrm{in}}}{L} & 0
\end{array}\right] \\
\boldsymbol{n}^{T} \lim _{\boldsymbol{t} \uparrow t_{\Sigma}} \boldsymbol{f}_{+}(\boldsymbol{x}(t)) & =\frac{x_{2}\left(t_{\Sigma}\right)-\frac{x_{1}\left(t_{\Sigma}\right)}{R}}{C} .
\end{aligned}
$$

Since we are considering a period-1 orbit, it suffices to consider $t$ varying from 0 to $T$ in (32), which gives

$$
\begin{aligned}
\frac{\partial h(\boldsymbol{x}(t), t)}{\partial t} & =\frac{\partial\left(x_{1}(t)-V_{\mathrm{ref}}-\frac{T V_{L}+\left(V_{U}-V_{L}\right) t}{A T}\right)}{\partial t} \\
& =-\frac{V_{U}-V_{L}}{A T} .
\end{aligned}
$$

${ }^{2}$ On this point some caution has to be exercised when considering specific periodic orbits, because di Bernardo et al. [33] have shown that in this system there can be orbits that slide along the switching surface. To use the concept of the saltation matrix to such orbits, care must be taken for the correct definition of the vector field after the switching. The case of discontinuous conduction mode falls in this category, and that analysis will be presented in a later publication. 
Hence, (26) is calculated as

$$
\boldsymbol{S}=\left[\begin{array}{cc}
\frac{1}{i} & 0 \\
\frac{\frac{V_{\text {in }}}{i}}{R} & 1
\end{array}\right] .
$$

Now, we need the state transition matrices for the two subsystems. The state transition matrix during the first time interval (i.e., when the switch is blocking) is given by $\boldsymbol{\Phi}_{1}\left(t_{\Sigma}, 0\right)=$ $e^{\mathbf{A}_{s} t_{\Sigma}}$. The state transition matrix for the second interval is given by $\boldsymbol{\Phi}_{2}\left(T, t_{\Sigma}\right)=e^{\mathbf{A}_{s}\left(T-t_{\Sigma}\right)}$, as the subsystems are linear time invariant. Knowing $S$, it is thus possible to calculate the monodromy matrix $\boldsymbol{\Phi}\left(t_{0}+T, t_{0}, \boldsymbol{x}_{0}\right)$ using the composition (27). At this point, it has to be noted that there is one more switching at $t=T$ at the end of the clock cycle, which will need another saltation matrix. It happens to be the identity matrix, since at this point $h$ is discontinuous and hence $\mathrm{d} h / \mathrm{d} t=\infty$. Furthermore, at this point, there is a forced switching, i.e., both the original and the perturbed orbits are forced to cross the switching manifold at the same time, and hence there is no need for a saltation matrix to describe the switching at $t=T$.

Finally, we need to substitute the numerical values of the state vector at the switching instant. Because of the transcendental form of the equations involved, the switching instant within the periodic cycle cannot be obtained explicitly. Following [8], it can however be obtained semi-analytically as follows.

Let the duty ratio be $d=t_{\mathrm{on}} / T=\left(T-t_{\Sigma}\right) / T$. For our convenience, we define the ratio $d^{\prime}=1-d=\left(T-t_{\text {on }}\right) / T$, $\boldsymbol{\Phi}_{1}\left(d^{\prime} T\right)=\boldsymbol{\Phi}_{1}\left(d^{\prime} T, 0\right)$, and $\boldsymbol{\Phi}_{2}(T)=\boldsymbol{\Phi}_{2}\left(T, d^{\prime} T\right)$. The value of the state vector at $t=t_{\Sigma}$ is

$$
\boldsymbol{x}\left(d^{\prime} T\right)=\boldsymbol{\Phi}_{1}\left(d^{\prime} T\right) \boldsymbol{x}(0) .
$$

The state vector when $t=T$ is given by

$$
\boldsymbol{x}(T)=\boldsymbol{x}(0)=\boldsymbol{\Phi}_{2}(T) \boldsymbol{x}\left(d^{\prime} T\right)+\int_{d^{\prime} T}^{T} e^{\mathbf{A}_{s}(T-\tau)}\left[\begin{array}{c}
0 \\
\frac{V_{\text {in }}}{L}
\end{array}\right] \mathrm{d} \tau \text {. }
$$

By eliminating $\boldsymbol{x}\left(d^{\prime} T\right)$ from (38) with (37), we get

$$
\begin{aligned}
\boldsymbol{x}(0)= & \boldsymbol{\Phi}_{2}(T) \boldsymbol{\Phi}_{1}\left(d^{\prime} T\right) \boldsymbol{x}(0) \\
& +\int_{d^{\prime} T}^{T} e^{\mathbf{A}_{s}(T-\tau)}\left[\begin{array}{c}
0 \\
\frac{V_{\text {in }}}{L}
\end{array}\right] \mathrm{d} \tau \\
= & {\left[\boldsymbol{I}-e^{\mathbf{A}_{s} T}\right]^{-1} \times \int_{d^{\prime} T}^{T} e^{\mathbf{A}_{s}(T-\tau)}\left[\begin{array}{c}
0 \\
\frac{V_{\text {in }}}{L}
\end{array}\right] \mathrm{d} \tau . }
\end{aligned}
$$

From the hypersurface, we have

$$
x_{1}\left(d^{\prime} T\right)=V_{\text {ref }}+\frac{V_{L}+\left(V_{U}-V_{L}\right) d^{\prime}}{A}
$$

and from (37) we have

$$
x_{1}\left(d^{\prime} T\right)=\left[\begin{array}{ll}
1 & 0
\end{array}\right] e^{\mathbf{A}_{s} d^{\prime} T} \boldsymbol{x}(0) .
$$

Hence

$$
\left[\begin{array}{ll}
1 & 0
\end{array}\right] e^{\mathbf{A}_{s} d^{\prime} T} \boldsymbol{x}(0)-V_{\mathrm{ref}}-\frac{V_{L}+\left(V_{U}-V_{L}\right) d^{\prime}}{A}=0 .
$$

After substituting $\boldsymbol{x}(0)$ from (39), (41) can be solved numerically with the Newton-Raphson method to obtain the value of $d^{\prime}$ for the periodic orbit.
TABLE I

SALTATION AND MONODROMY MATRICES FOR VARIOUS INPUT VOLTAGES

\begin{tabular}{|c|c|c|c|c|c|}
\hline$V_{i n}, \mathrm{~V}$ & $d^{\prime}$ & \multicolumn{2}{|c|}{ Saltation matrix } & Monodromy matrix & Floquet mulipliers \\
\hline 14 & 0.1559 & {$\left[\begin{array}{cc}1 & 0 \\
-0.4072 & 1\end{array}\right]$} & {$\left[\begin{array}{ll}-1.6832 & 3.7123 \\
-0.3780 & 0.4301\end{array}\right]$} & $-0.6265 \pm 0.5354 j$ \\
\hline 20 & \multirow{2}{*}{0.4024} & {$\left[\begin{array}{cc}1 & 0 \\
-0.4262 & 1\end{array}\right]$} & {$\left[\begin{array}{ll}-1.0020 & 0.8115 \\
-0.3655 & -0.3818\end{array}\right]$} & $-0.6919 \pm 0.4477 j$ \\
\hline 24 & 0.4993 & {$\left[\begin{array}{cc}1 & 0 \\
-0.4639 & 1\end{array}\right]$} & {$\left[\begin{array}{cc}-0.8238 & 0.0131 \\
-0.3825 & -0.8184\end{array}\right]$} & $-0.8211 \pm 0.0708 j$ \\
\hline 25 & 0.5187 & {$\left[\begin{array}{cc}1 \\
-0.4744 & 1\end{array}\right]$} & {$\left[\begin{array}{ll}-0.7919 & -0.1323 \\
-0.3878 & -0.9225\end{array}\right]$} & $\left\{\begin{array}{l}-0.6214 \\
-1.0929\end{array}\right\}$ \\
\hline
\end{tabular}

\section{Stability ANALYSis of THE BUCK CONVERTER}

For $V_{\text {in }}=24 \mathrm{~V}$ and $V_{\text {ref }}=11.3 \mathrm{~V}$, the Newton-Raphson solution obtained $d^{\prime}=0.4993$. Then, from (39) and (40), we get

$$
\boldsymbol{x}(0)=\left[\begin{array}{c}
12.0222 \\
0.6065
\end{array}\right] \quad \boldsymbol{x}\left(d^{\prime} T\right)=\left[\begin{array}{c}
12.0139 \\
0.4861
\end{array}\right] .
$$

The saltation matrix is calculated as

$$
\boldsymbol{S}=\left[\begin{array}{cc}
1 & 0 \\
-0.4639 & 1
\end{array}\right]
$$

The state matrix (which is the same before and after the jump for the buck converter) is given by

$$
\mathbf{A}_{s}=\left[\begin{array}{cc}
967.12 & 21276.6 \\
-50 & 0
\end{array}\right]
$$

The state transition matrices for the two pieces of the orbit are

$$
\begin{gathered}
\boldsymbol{\Phi}\left(d^{\prime} T, 0\right)=e^{\mathbf{A}_{s} d^{\prime} T}=\left[\begin{array}{cc}
0.8058 & 3.8366 \\
-0.0090 & 0.9802
\end{array}\right] \\
\boldsymbol{\Phi}\left(T, d^{\prime} T\right)=e^{\mathbf{A}_{s} d T}=\left[\begin{array}{cc}
0.8052 & 3.8468 \\
-0.0090 & 0.9800
\end{array}\right]
\end{gathered}
$$

respectively. Hence, the monodromy matrix is

$$
\boldsymbol{\Phi}(T, 0, x(0))=\left[\begin{array}{cc}
-0.8238 & 0.0131 \\
-0.3825 & -0.8184
\end{array}\right] .
$$

The eigenvalues of (43) are $-0.8211 \pm 0.0708 j$, implying that at the above parameter values the system is stable. This is in agreement with the experimental and numerical observations of the system behavior, reported in Section VII, as well as with those reported in earlier studies of the same system [7]. To further confirm the results obtained with the new method of analysis, the Floquet multipliers (or the eigenvalues of the monodromy matrix) were calculated for values of the input voltage $V_{\text {in }}$ ranging from 14 to $25 \mathrm{~V}$. Table I shows the calculated values of the saltation matrix, the monodromy matrix, and its Floquet multipliers. The locus of these eigenvalues is shown in Fig. 6. It shows that eigenvalues first became real for a specific parameter value, and then one of the eigenvalues went out of the unit circle through the negative real line, marking the onset of instability through a period-doubling bifurcation.

It is interesting to point out that the change in the value of $d^{\prime}$ for $V_{\text {in }}$ varied from $24 \mathrm{~V}$ to $25 \mathrm{~V}$ is approximately -0.0195 , which will only slightly change the matrices $\boldsymbol{\Phi}_{2}(T), \boldsymbol{\Phi}_{1}\left(d^{\prime} T\right)$ 


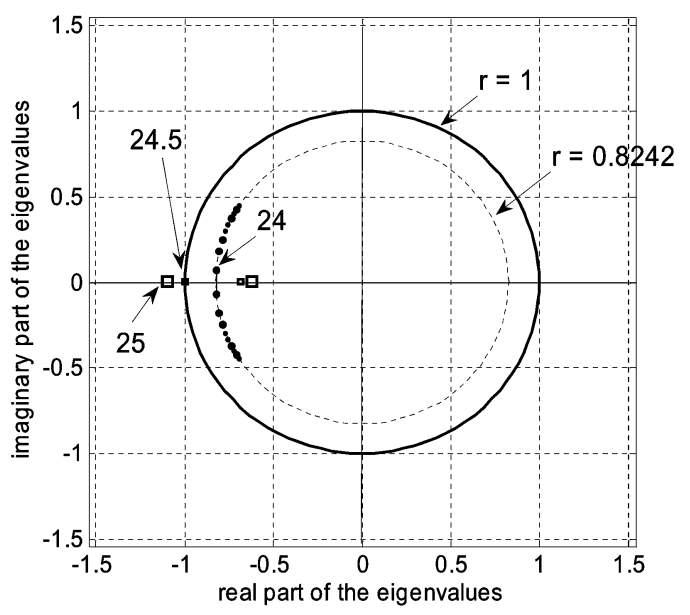

Fig. 6. Eigenvalue loci for $V_{\text {in }} \in[14 \mathrm{~V}, 25 \mathrm{~V}]$. Squares indicate an unstable system, and solid circles represent a stable system.

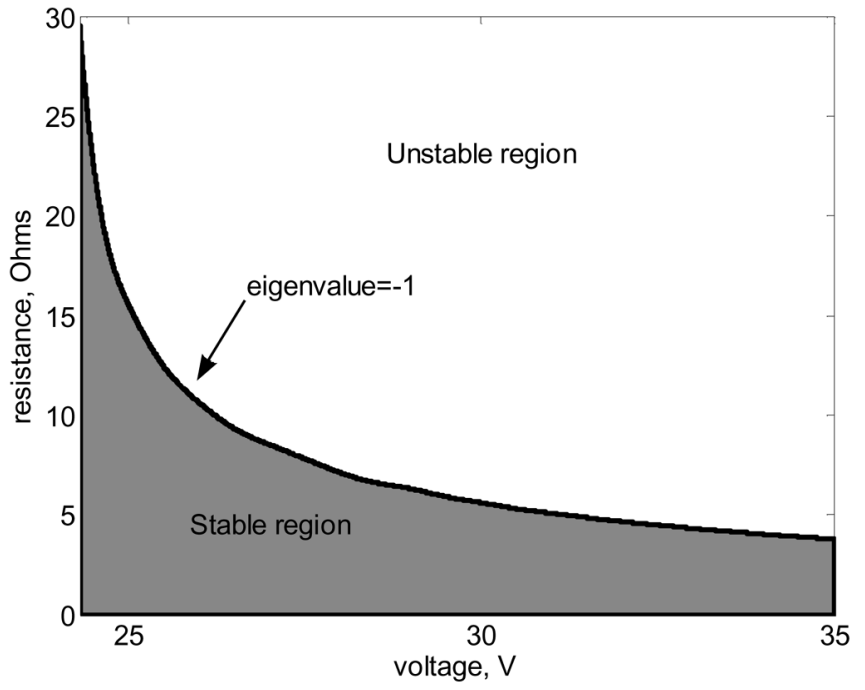

Fig. 7. Region of stability of the period- 1 orbit in the $V_{\text {in }}-R$ parameter space. The contour is obtained using the condition that the lower eigenvalue of the monodromy matrix is equal to -1 .

and yet the system becomes unstable. The answer to this surprising circuit behavior lies in the corresponding change of the saltation matrix, given by

$$
\left[\begin{array}{cc}
0 & 0 \\
0.0105 & 0
\end{array}\right]
$$

which causes an eigenvalue of the monodromy matrix to go out of the unit circle.

This provides a simple way for the circuit designer to choose the parameters. Given certain specifications of the input and output voltages and power throughput, the designer would first roughly set a range of parameters in the conventional way, based on the averaged model to get the desired slow time scale stability and transient performance. But this will not guarantee that the system will be stable on a fast time scale when variable parameters like input voltage and load resistance fluctuate. In order to ensure that, it will be necessary to calculate the range of the variable parameters like $V_{\text {in }}$ and $R$ for which the period-1 orbit will remain stable. A representative parameter space diagram, with other parameters fixed at values given in the caption of Fig. 4, is shown in Fig. 7. The designer will have to ensure that the

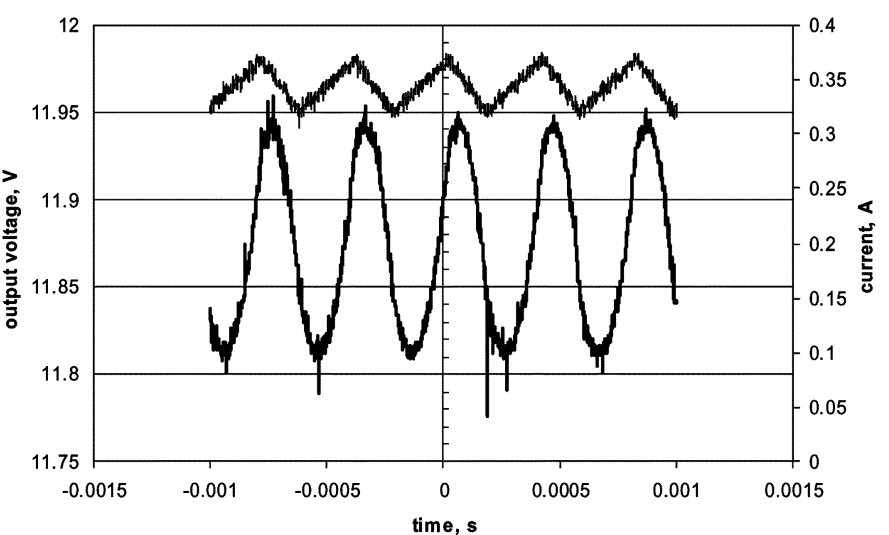

(a)

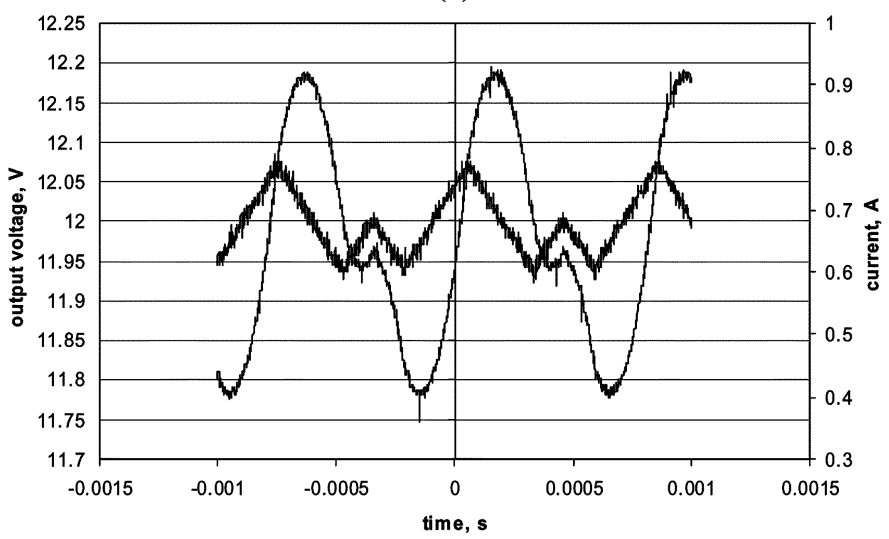

(b)

Fig. 8. Experimental waveforms for the capacitor voltage and inductor current (a) for $V_{\text {in }}=20 \mathrm{~V}$ and (b) for $V_{\text {in }}=25 \mathrm{~V}$.

external parameters remain within the shaded region of the parameter space.

\section{EXPERIMENTAL RESULTS}

To further validate these results, experimental tests were carried out. Results are presented in Fig. 8 which show that an instability does occur in the period-1 orbit before $V_{\text {in }}=25 \mathrm{~V}$, which results in the birth of the period- 2 orbit. The results are in total agreement with the theoretical predictions presented in Fig. 6 and Table I.

One particular strength of the method developed in this paper is that the solution of (41) does not distinguish between a stable orbit and an unstable orbit. Therefore, unstable periodic orbits can be detected and their Floquet exponents can be calculated. An example of this is presented in Fig. 9, where the unstable period-1 orbit coexisting with the stable period- 2 orbit for $V_{\text {in }}=$ $25 \mathrm{~V}$ is shown. It is known that such unstable periodic orbits, though invisible as far as asymptotic behavior is concerned, can influence the bifurcation sequence through border collision bifurcation or interior crisis.

\section{FURTHER APPLICATIONS}

Here, we will apply the methodology developed in this paper to explain some empirical observations reported recently, and to propose a new controller that delays the bifurcation and can allow the nominal period-1 orbit to continue for a larger parameter range. 


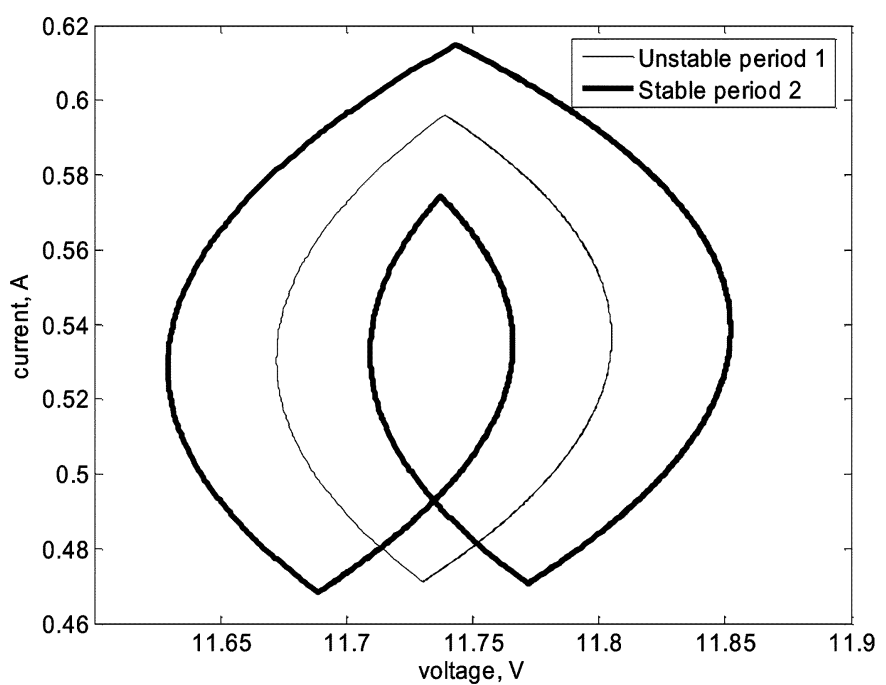

Fig. 9. Stable and unstable periodic orbits of the buck converter for $V_{\text {in }}=$ $25 \mathrm{~V}$.

\section{A. Explanation of Intermittent Subharmonic Windows}

There have been some recent reports [21], based on empirical observations from simulation as well as experiments, that the system exhibits some peculiar intermittent subharmonic behavior when the reference signal is coupled with a small-amplitude time-varying signal. Such spurious additive signal may represent coupling with the environment, and so it is a matter of great importance to understand why they occur.

Let us first consider sinusoidal additive signal, so that $V_{\text {ref }}$ takes the form $V_{\text {ref }}\left(1+a \sin \omega_{s} t\right)$, where $\omega_{s}$ may be different from $\omega=2 \pi / T$. It has been shown [21] that, in that case, intermittent subharmonic windows appear. If we assume $\omega_{s}=\omega$, the length of the specific subharmonic window is extended to infinity, and so it becomes a steady-state behavior. Then, we use the eigenvalues of the monodromy matrix to predict the change of stability properties.

Assuming $\omega_{s}=\omega$, the scalar equation $h(\boldsymbol{x}(t), t)=0$ that describes the hypersurface is

$x_{1}-V_{\text {ref }}-V_{\text {ref }} a \sin \omega t-\frac{T V_{L}+\left(V_{U}-V_{L}\right) t}{A T}=0, \quad t \in(0, T)$.

In this case, (41) takes the form

$$
\begin{gathered}
{\left[\begin{array}{ll}
1 & 0
\end{array}\right] e^{\mathbf{A}_{s} d^{\prime} T}\left(\left[I-e^{\mathbf{A}_{s} T}\right]^{-1} \times \int_{d^{\prime} T}^{T} e^{\mathbf{A}_{s}(T-\tau)}\left[\begin{array}{c}
0 \\
\frac{V_{\text {in }}}{L}
\end{array}\right] \mathrm{d} \tau\right)} \\
-V_{\text {ref }}-V_{\text {ref }} a \sin \left(\omega d^{\prime} T\right)-\frac{V_{L}+\left(V_{U}-V_{L}\right) d^{\prime}}{A}=0 .
\end{gathered}
$$

The duty cycles and the corresponding values of the state vector at $t=0$ and $t=d^{\prime} T$ (for the period 1 cycle) are shown in Table II.

Studying the results presented in Table II, it can be seen that the duty cycle and hence the vector fields at $t=0$ and $t=d^{\prime} T$ remain almost unchanged. Furthermore, $h\left(x\left(d^{\prime} T\right), d^{\prime} T\right)$ does not change much since the value of $V_{\text {ref }} a \sin \left(\omega d^{\prime} T\right)$ is almost zero. This could lead to the wrong conclusion that the additive signal makes no change in the stability status, which contradicts the results reported in [21]. The underlying situation becomes
TABLE II

VALUES OF $d^{\prime}$ AND THE STATE VECTOR FOR $V_{\text {in }}=24 \mathrm{~V}$ AND VARIOUS VALUES OF $a$

\begin{tabular}{|c|c|cc|c|}
\hline$a$ & $d^{\prime}$ & $\boldsymbol{x}(0)$ & $\boldsymbol{x}\left(d^{\prime} T\right)$ \\
\hline 0 & 0.4993 & {$\left[\begin{array}{ll}12.0222 & 0.6065\end{array}\right]^{T}$} & {$\left[\begin{array}{ll}12.0139 & 0.4861\end{array}\right]^{T}$} \\
\hline 0.0001 & 0.4993 & {$\left[\begin{array}{ll}12.0222 & 0.6065\end{array}\right]^{T}$} & {$\left[\begin{array}{ll}12.0139 & 0.4861\end{array}\right]^{T}$} \\
\hline 0.0002 & 0.4993 & {$\left[\begin{array}{ll}12.0222 & 0.6065\end{array}\right]^{T}$} & {$\left[\begin{array}{ll}12.0139 & 0.4861\end{array}\right]^{T}$} \\
\hline 0.0003 & 0.4993 & {$\left[\begin{array}{ll}12.0222 & 0.6065\end{array}\right]^{T}$} & {$\left[\begin{array}{ll}12.0139 & 0.4861\end{array}\right]^{T}$} \\
\hline
\end{tabular}

TABLE III

EIGENVALUES OF THE MONODROMY MATRIX FOR INCREASING VALUES OF $a$

\begin{tabular}{|c|c|}
\hline$a$ & Eigenvalues \\
\hline 0 & $-0.8211 \pm 0.0708 j$ \\
\hline 0.0001 & {$\left[\begin{array}{ll}-0.9468 & -0.7174\end{array}\right]^{T}$} \\
\hline 0.0002 & {$\left[\begin{array}{ll}-1.0216 & -0.6648\end{array}\right]^{T}$} \\
\hline 0.0003 & {$\left[\begin{array}{ll}-1.0804 & -0.6286\end{array}\right]^{T}$} \\
\hline
\end{tabular}

clear only when we obtain the eigenvalues of the monodromy matrix, as given in Table III. It shows that for the amplitude of the additive signal as low as $a=0.0002$, the monodromy matrix has an eigenvalue outside the unit circle and hence the period-1 orbit is unstable (a stable period-2 orbit is born out of a flip bifurcation).

To understand why a flip bifurcation takes place for that value, while the duty cycle remains constant, it is necessary to study the saltation matrix. We reproduce the expression here

$$
\boldsymbol{S}=\boldsymbol{I}+\frac{\left(\lim _{t \downarrow t_{\Sigma}}(\boldsymbol{f}(\boldsymbol{x}(t)))-\lim _{t \uparrow t_{\Sigma}}(\boldsymbol{f}(\boldsymbol{x}(t)))\right) \boldsymbol{n}^{T}}{\boldsymbol{n}^{T} \lim _{t \uparrow t_{\Sigma}}(\boldsymbol{f}(\boldsymbol{x}(t)))+\left.\frac{\partial h}{\partial t}(\boldsymbol{x}(t), t)\right|_{t=t_{\Sigma}}} .
$$

The only component that changed with the addition of $V_{\mathrm{ref}} a \sin (\omega t)$ is the last term in the denominator, since the duty cycle remained practically unchanged. This term is defined as

$$
\frac{\partial h}{\partial t}(x(t), t)=-V_{\text {ref }} a \omega \cos \omega t-\frac{V_{U}-V_{L}}{A T}
$$

which is obviously different from that without the additive signal. Add to this the observation that the value of $V_{\text {ref }} a \sin \left(\omega d^{\prime} T\right)$ is very small, which implies that the value of $-V_{\text {ref }} a \omega \cos (\omega t)$ will be large at $t=t_{\Sigma}$. Therefore, there will be a large influence on the value of $\partial h / \partial t\left(x(t), t_{\Sigma}\right)$ and therefore on the saltation matrix. This greatly influences the monodromy matrix as it can be seen in Table III, and renders the orbit unstable.

\section{B. Development of a Control Strategy}

Based on the observation in [21], a chaos control scheme has been recently proposed in [22]. With the approach proposed in this paper, we are now in a position to understand why this chaos controlling scheme works.

Since a positive value of $a$ pushes one eigenvalue of the monodromy matrix towards -1 , a negative value of $a$ (i.e., a phase shift of $180^{\circ}$ ) is expected to have a reverse effect. Hence, if we have an uncoupled buck converter which exhibits a period doubling at a specific parameter value, a small time varying 


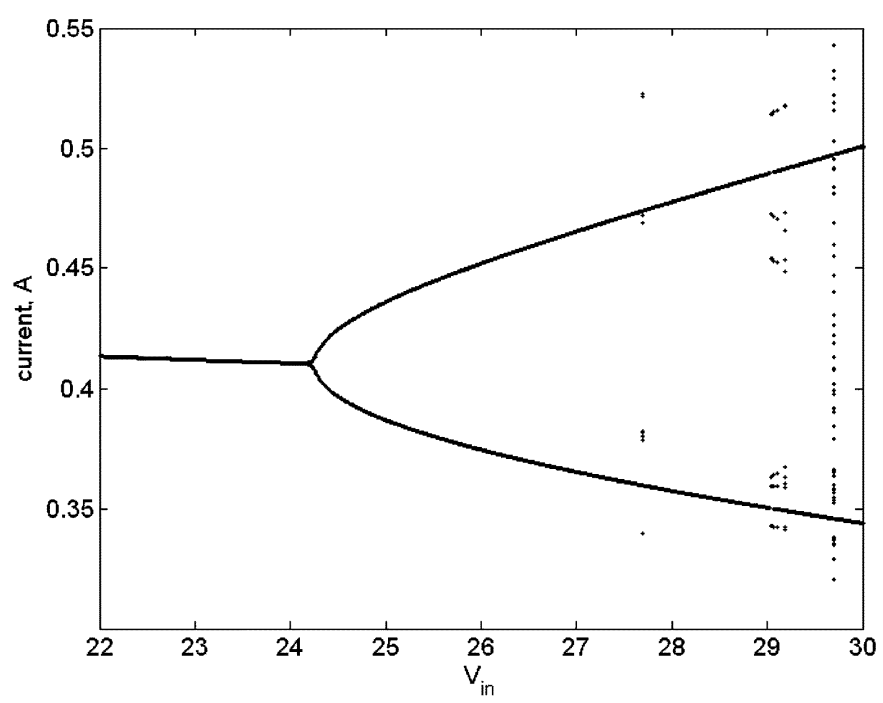

Fig. 10. Bifurcation diagram of the system.

signal added to $V_{\text {ref }}$ will force the period-1 limit cycle to become stable once again. This is feasible since the period doubling did not destroy the period-1 orbit but simply changed its stability properties.

Our approach has more to offer than just explanation of empirical observations. We can now "optimally" choose the strength of the additive sine wave. One strategy may be to keep the magnitude of the eigenvalues exactly the same as that for the stable periodic orbit obtained for low values of $V_{\text {in }}$. It can be seen in Fig. 6 that, for low values of $V_{\text {in }}$, the eigenvalues lie on a circle of radius 0.8241 . To obtain the value of $a$, we used a Newton-Raphson method to place the eigenvalues of the monodromy matrix on that circle by solving the equation

$$
|\operatorname{eig}(\boldsymbol{\Phi}(T, 0, \boldsymbol{x}(0)))|-0.8242=0 .
$$

The results of this algorithm for various values of $V_{\text {in }}$ are shown in Fig. 11. Based on these values, we created a third-order polynomial expression

$a=-2.2 \times 10^{-6} V_{\text {in }}^{3}+1.5 \times 10^{-4} V_{\text {in }}^{2}-3.7 \times 10^{-3} V_{\text {in }}+3.3^{-2}$

that matches the graph of Fig. 11 over a large range of the input voltage.

By using this equation, we propose an improvement of the chaos controlling scheme that appears in [22]. We create a supervising controller whose task is to change the value of $a$, and then another controller adds the signal a $V_{\text {ref }} \sin \omega t$ to the reference input value (see Fig. 12).

The output voltage response to a step change in input voltage $(24-30 \mathrm{~V})$ is shown in Fig. 13. Without the supervising controller, it would have resulted in a period-2 subharmonic oscillatory mode, as can be seen in the bifurcation diagram given in Fig. 10. Note that, following the transient, the system briefly goes into period- 2 mode, but is quickly corrected by the supervising controller to reestablish the period-1 operation. We have found that this controller is robust to load resistance changes also. Further research is underway to avoid the offline calculation of the values of $a$ by using artificial intelligence methods

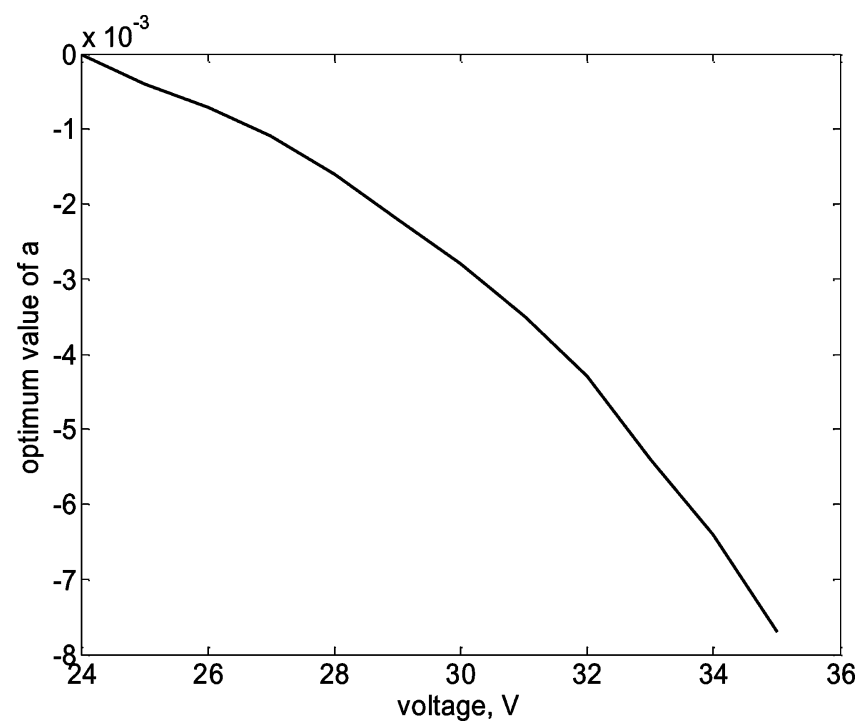

Fig. 11. Optimum values of $a$ for varying $V_{\text {ref }}$.

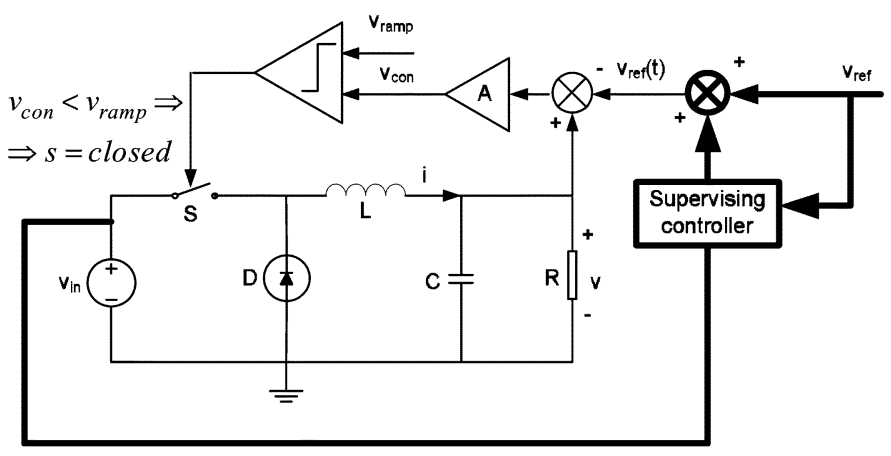

Fig. 12. Supervising controller.

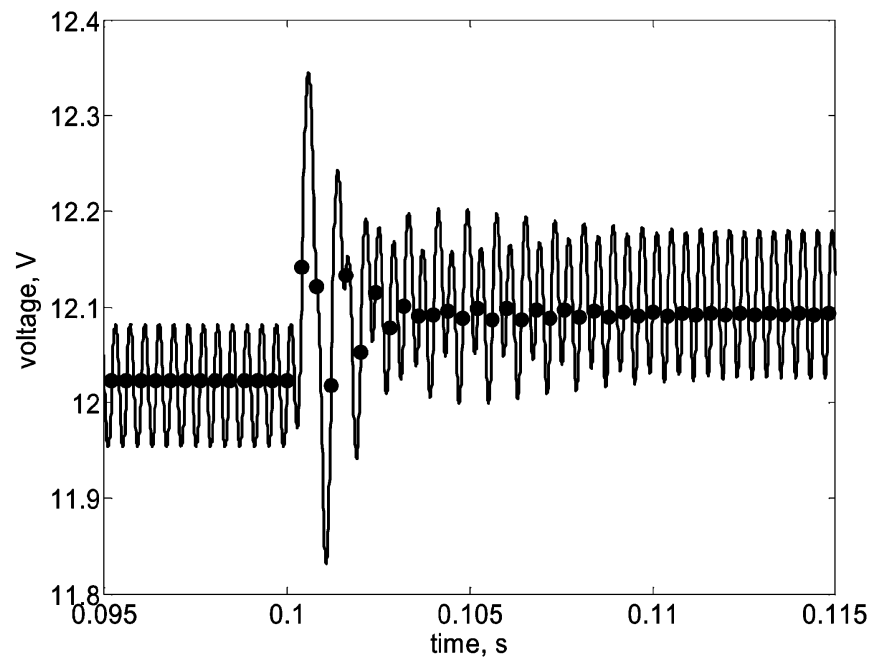

Fig. 13. Voltage response to a step change in input voltage from 24 to $30 \mathrm{~V}$, shown along with the stroboscopic sampling points.

like adaptive critical control. It has to be noted that the sinusoidal signal used here has zero phase at the clock instant, which works quite well if the duty cycle is close to $50 \%$. In a general case we will choose the zero crossing of the imposed sinusoid to coincide approximately with the switching instant, which can be done if an approximate value of the duty ratio is known. This will ensure that the switching instant (and hence the duty ratio) 
is not affected by the sinusoidal signal, but the derivative of the switching manifold with respect to time will be maximum in magnitude at the switching instant.

\section{CONCLUSION}

Following Filippov's approach, we have developed a powerful technique to determine the stability of periodic limit cycles in power electronic circuits. The method is quite suitable for stability analysis of the vast majority of power electronic systems whose Poincaré map cannot be determined in closed form.

In this method, the fundamental solution matrix over a complete cycle — called the monodromy matrix-is determined by using the state transition matrices for the segments of the orbit lying in the individual subsystems, and the transition matrix across the switching boundaries-called the saltation matrix. We have illustrated the method by applying it to the voltage mode controlled buck converter, yielding valuable insights into issues related to the stability of the nominal period-1 orbit. The saltation matrix concept can also be fruitfully used to model the evolution of perturbations in case of situations like thyristor switch off and diode switch on, where the system dimension changes [3] across the switching manifold. We have shown that the saltation matrix is principally responsible in the determination of the system's stability. By this method, one can calculate the range of the variable parameters like the input voltage and load resistance for which the period-1 orbit will be stable.

We have then used this approach to explain the recent observation that spurious sinusoidal additive signals cause intermittent subharmonic behavior in such converters. Finally, we have proposed a supervising controller that can extend the parameter range over which the period-1 operation of the converter remains stable.

\section{ACKNOWLEDGMENT}

The authors would like to thank Dr. R. I. Leine (Centre of Mechanics, Institute of Mechanical Systems, ETH Zurich, Switzerland) for his valuable and highly appreciated advice and comments during the preparation of this paper.

\section{REFERENCES}

[1] R. D. Middlebrook and S.Ćuk, "A general unified approach to modeling switching converter power stages," in Proc. IEEE Power Electron. Specialists' Conf., Cleveland, OH, 1976, pp. 18-34.

[2] J. G. Kassakian, M. F. Schlecht, and G. C. Verghese, Principles of Power Electronics. Reading, MA: Addison-Wesley, 1991.

[3] S. Banerjee and G. C. Verghese, Eds., Nonlinear Phenomena in Power Electronics: Attractors, Bifurcations, Chaos, and Nonlinear Control. New York: IEEE Press, 2001.

[4] C. K. Tse, Complex Behavior of Switching Power Converters. Boca Raton, FL: CRC, 2003.

[5] J. H. B. Deane and D. C. Hamill, "Instability, subharmonics, and chaos in power electronics circuits," IEEE Trans. Power Electron., vol. 5, no. 3, pp. 260-268, Jul. 1990.

[6] K. Chakrabarty, G. Poddar, and S. Banerjee, "Bifurcation behaviour of the buck converter," IEEE Trans. Power Electron., vol. 11, no. 2, pp. 439-447, May 1996.

[7] E. Fossas and G. Olivar, "Study of chaos in the buck converter," IEEE Trans. Circuits Syst. I, Fundam. Theory Appl., vol. 43, no. 1, pp. 13-25, Jan. 1996.
[8] G. C. Verghese, M. E. Elbuluk, and J. G. Kassakian, "A general approach to sampled-data modeling for power electronic circuits," IEEE Trans. Power Electron., vol. PE-1, no. 1, pp. 76-89, 1986.

[9] G. C. Verghese, "Averaged and sampled-data models for current-mode control: a reexamination," in Proc. Power Electronic Specialists' Conf., 1989, pp. 484-491.

[10] D. C. Hamill and J. H. B. Deane, "Modeling of chaotic dc-dc converters by iterated nonlinear mappings," IEEE Trans. Power Electron., vol. 7, no. 1, pp. 25-36, Jan. 1992.

[11] M. D. Bernardo and F. Vasca, "Discrete-time maps for the analysis of bifurcations and chaos in dc/dc converters," IEEE Trans. Circuits Syst. I, Fundam. Theory Appl., vol. 47, no. 2, pp. 130-143, Feb. 2000.

[12] J. H. B. Deane and D. C. Hamill, "Chaotic behaviour in current-mode controlled dc-dc converter," Electron. Lett., vol. 27, pp. 1172-1173, Jun. 1991.

[13] S. Banerjee and K. Chakrabarty, "Nonlinear modeling and bifurcations in the boost converter," IEEE Trans. Power Electron., vol. 13, no. 3, pp. 252-260, Jul. 1998.

[14] M. A. Aizerman and F. R. Gantmakher, "On the stability of periodic motions," (in Russian) J. Appl. Math. Mechan., pp. 1065-1078, 1958.

[15] A. F. Filippov, Differential Equations With Discontinuous Righthand Sides. Dordrecht, The Netherlands: Kluwer, 1988.

[16] R. I. Leine, D. H. V. Campen, and B. L. V. de Vrande, "Bifurcations in nonlinear discontinuous systems," Nonlinear Dynam., vol. 23, pp. $105-164,2000$

[17] R. I. Leine and H. Nijmeijer, Dynamics and Bifurcations of NonSmooth Mechanical Systems. Berlin, Germany: Springer-Verlag, 2004.

[18] M. M. Polycarpou and P. A. Ioannou, "On the existence and uniqueness of solutions in adaptive control systems," IEEE Trans. Autom. Control, vol. 38, no. 2, pp. 474-479, Mar. 1993.

[19] D. Shevitz and B. Paden, "Lyapunov stability theory of nonsmooth systems automatic control," IEEE Trans. Autom. Control, vol. 39, no. 6, pp. 1910-1914, Sep. 1994.

[20] B. E. Paden and S. S. Sastry, "A calculus for computing Filippov's differential inclusion with application to the variable structure control of robot manipulators," IEEE Trans. Circuits Syst., vol. CAS-34, no. 1, pp. 73-82, Jan. 1987.

[21] C. K. Tse, Y. Zhou, F. C. M. Lau, and S. S. Qiu, "Intermittent chaos in switching power suplies due to unintended coupling of spurious signals," in Proc. Power Electron. Specialists' Conf., Acapulco, Mexico, Jun. 2003, pp. 642-647.

[22] Y. Zhou, C. K. Tse, S. S. Qiu, and F. C. M. Lau, "Applying resonant parametric perturbation to control chaos in the buck dc/dc converter with phase shift and frequency mismatch considerations," Int. J. Bifurc. Chaos, vol. 13, pp. 3459-3471, Nov. 2003.

[23] H. K. Khalil, Nonlinear Systems. Upper Saddle River, NJ: PrenticeHall, 2002.

[24] I. A. Hiskens, "Stability of limit cycles in hybrid systems," in Proc. 34th Hawaii Int. Conf. Syst. Sci., 2001, p. 2022.

[25] Y. A. Kuznetsov, Elements of Applied Bifurcation Theory. Berlin, Germany: Springer-Verlag, 2004.

[26] I. A. Hiskens and M. A. Pai, "Trajectory sensitivity analysis of hybrid systems," IEEE Trans. Circuits Syst. I, Fundam. Theory Appl., vol. 47, no. 2, pp. 204-220, Feb. 2000.

[27] C.-C. Fang and E. H. Abed, "Sampled-data modeling and analysis of closed-loop PWM dc-dc converters," in Proc. IEEE Int. Symp. Circuits Syst., 1999, vol. 5, pp. 110-115.

[28] C.-C. Fang and E. H. Abed, "Sampled-data modelling and analysis of the power stage of PWM dc-dc converters," Int. J. Electron., vol. 88, pp. 347-369, Mar. 2001.

[29] O. Dranga, B. Buti, I. Nagy, and H. Funato, "Stability analysis of nonlinear power electronic systems utilizing periodicity and introducing auxiliary state vector," IEEE Trans. Circuits Syst. I, Reg. Papers, vol. 52, no. 1, pp. 168-178, Jan. 2005.

[30] Y. Ma, H. Kawakami, and C. K. Tse, "Bifurcation analysis of switched dynamical systems with periodically moving borders," IEEE Trans. Circuits Syst. I, Reg. Papers, vol. 51, no. 6, pp. 1184-1193, Jun. 2004.

[31] A. B. Nordmark, "Universal limit mapping in grazing bifurcations," Phys. Rev. E, vol. 55, pp. 266-270, Jan. 1997.

[32] H. J. Dankowicz and P. T. Piiroinen, "Exploiting discontinuities for stabilization of recurrent motions," Dynamical Syst., vol. 17, pp. 317-342, Dec. 2002.

[33] M. D. Bernardo, F. Garofalo, L. Glielmo, and F. Vasca, "Switchings, bifurcations and chaos in dc-dc converters," IEEE Trans. Circuits Syst. I, Fundam. Theory Appl., vol. 45, no. 2, pp. 133-141, Feb. 1998. 


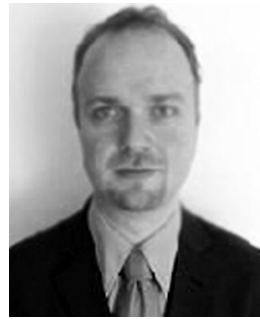

Damian Giaouris (M'04) was born in Munich, Germany, in 1976. He received the Diploma of automation engineering from the Technological Educational Institute of Thessaloniki, Thessaloniki, Greece, in 2000, and the M.Sc. degree in automation and control the Ph.D. degree in the area of control and stability of induction machine drives from University of Newcastle, Newcastle upon Tyne, U.K., in 2001 and 2004, respectively.

His research interests involve advanced nonlinear control, estimation, digital signal processing methods applied to electric drives and nonlinear phenomena in power electronic converters. He is currently a lecturer in Control Systems with University of Newcastle.

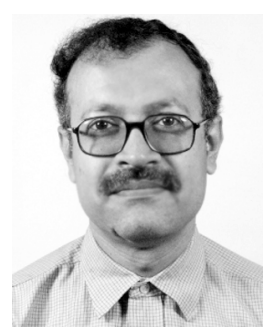

Soumitro Banerjee (SM'02) was born in 1960 $\mathrm{He}$ received the B.E. degree from the Bengal Engineering College (Calcutta University), Calcutta, India, in 1981 and the M.Tech. and Ph.D. degrees from the Indian Institute of Technology (IIT), Delhi, in 1983 and 1987, respectively.

He has been a member of the faculty of the Department of Electrical Engineering, IIT Kharagpur, since 1986. His areas of interest are the nonlinear dynamics of power electronic circuits and systems and bifurcation theory for nonsmooth systems. He has published three books: Nonlinear Phenomena in Power Electronics (IEEE Press, 2001), Dynamics for Engineers (Wiley, 2005), and Wind Electrical Systems (Oxford University Press, 2005).

Dr. Banerjee is a Fellow of the Indian Academy of Sciences, the Indian National Academy of Engineering, and the Indian National Science Academy. $\mathrm{He}$ served as an Associate Editor for the IEEE TRANSACTIONS ON CIRCUITS AND SYSTEMS II-EXPRESS BRIEFS (2003-2005) and is currently an Associate Editor of the IEEE TRANSACTIONS ON CIRCUITS AND SYSTEMS I-REGULAR PAPERS. He was a recipient of the S. S. Bhatnagar Prize in 2003 and the Citation Laureate Award in 2004.

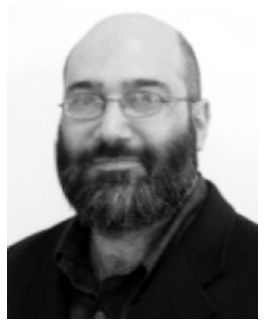

Bashar Zahawi (SM'04) received the B.Sc. and $\mathrm{Ph} . \mathrm{D}$. degrees in electrical and electronic engineering from the University of Newcastle, Newcastle upon Tyne, U.K., in 1983 and 1988, respectively.

From 1988 to 1993, he was a Design Engineer with Cortina Electric Company Ltd., a U.K. manufacturer of large ac variable speed drives and other power conversion equipment. In 1994, he was appointed as a Lecturer of electrical engineering with the University of Manchester, Manchester, U.K., and, in 2003, he joined the School of Electrical, Electronic and Computer Engineering, University of Newcastle, where he is currently the Director of Postgraduate Studies. His research interests include power conversion, variable speed drives, and the application of nonlinear dynamical methods to transformer and power electronic circuits.

Dr. Zahawi is aChartered Electrical Engineer in the U.K.

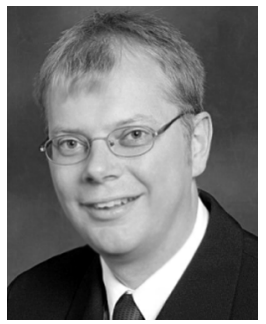

Volker Pickert (M'04) studied electrical and electronic engineering at the RWTH, Aachen, Germany. He received the Dipl.-Ing. degree from the University of Cambridge, Cambridge, U.K., in 1994 and the $\mathrm{Ph}$.D. degree in power electronics from University of Newcastle, Newcastle upon Tyne, U.K., in 1997.

After his doctoral studies, he was an Application Engineer with Semikron International for two years and for five years as a Project Manger for Volkswagen, where he was responsible for power electronic systems and electric drives for electric and hybrid electric vehicles. Since 2003, he has been a Senior Lecturer with University of Newcastle. His research interests are power electronics in automotive applications, thermal management, fault-tolerant power converters, and nonlinear controllers. 